\title{
The Role of Noise in Forming the Dynamics of a Quasiperiodic System
}

\author{
Natalia A. Khovanova*, Igor A. Khovanov*, Vadim S.Anishchenko* \\ and Peter V.E. McClintock ${ }^{\dagger}$ \\ * Department of Physics, Saratov State University, \\ Astrakhanskaya str. 83, 410026 Saratov, Russia \\ ${ }^{\dagger}$ Department of Physics, Lancaster University, Lancaster, LA1 $4 Y B, U K$
}

\begin{abstract}
The dynamical properties of the quasiperiodic logistic map with and without a very weak noise are compared, and the influence of noise on its strange nonchaotic attractor (SNA) is investigated. It is found that, in the presence of weak noise, the largest Lyapunov exponent gives misleading information about the dynamical properties of the attractor. We have shown that, in the presence of noise, the properties of strangeness and chaos are invariably associated, so that SNAs are not then observed during the transition to chaos from the torus.
\end{abstract}

During the last thirty years, quasiperiodic systems, i.e. those whose dynamics is formed by signals with two or more independent frequencies, have attracted the attention of scientists from many different fields. Such systems display interesting and often unexpected properties, one of which is the presence of strange nonchaotic attractors (SNAs) [1]. Before SNAs were discovered it was assumed that strange and chaotic were synonyms, even though they describe quite different features: the attractor's geometrical and dynamical properties respectively. An SNA is strange in that it has a geometrically complex structure (i.e. the attractor is not a finite set of points, and is not piecewise differentiable), and it is nonchaotic in that it exhibits no sensitivity to initial conditions. In quasiperiodic systems an SNA is usually observed as the intermediate stage in the evolution of the system from the torus to the chaotic regime [2].

In [2] and in most other papers on the subject, quasiperiodic systems are studied in the absence of noise, i.e. no account is taken of the fact that real macroscopic physical systems are always subject to random fluctuations. It is well known [3], however, that the interaction between the nonlinear deterministic part of a dynamical system and internal or external fluctuations can lead to a range of interesting and important phenomena. In this paper, we study the influence of noise on the properties of an SNA in a quasiperiodic system.

As the object of our investigations we have chosen the quasiperiodic logistic map:

CP502, Stochastic and Chaotic Dynamics in the Lakes: STOCHAOS,

edited by D. S. Broomhead, E. A. Luchinskaya, P.V. E. McClintock, and T. Mullin

(C) 2000 American Institute of Physics 1-56396-915-7/00/\$17.00 


$$
\begin{array}{r}
x_{n+1}=\alpha\left(1+\beta \cos \phi_{n}\right) x_{n}\left(1-x_{n}\right)+D \xi_{n}, \\
\phi_{n+1}=\phi_{n}+\rho \bmod 2 \pi,
\end{array}
$$

in which: $x_{n} \in[0,1] ; \rho=2 \pi \omega ; \xi_{n}$ is Gaussian noise of intensity $D^{2}$; and $\alpha, \beta, \omega$ are parameters. It describes the dynamics of flow under a two-frequency quasiperiodic force and, in the absence of noise, is the paradigm system for studying SNAs. By changing $\alpha$, we can observe in turn: the torus; an SNA; and a strange chaotic attractor. The route by which the SNA appears has been considered in detail in $[4,5]$. Following [4], we fix $\beta=0.01$ and $\omega=(\sqrt{5}-1) / 2$, and we designate $\alpha$ as the control parameter.

In the absence of noise [4] for $\alpha<3.52760$ a torus is observed; at $\alpha \approx 3.52760$ a collision occurs between the period- 2 repellor and the period- 4 attractor. The resultant fractal structure [4] means that for $\alpha>3.52760$ the attractor is strange. To confirm this picture, we have calculated (Fig. 1) the fractal dimension $D_{F}$, which defines the metric properties of an attractor in phase space [6]: for $\alpha>3.52760$, $D_{F}$ differs markedly from unity, implying strangeness of the attractor.

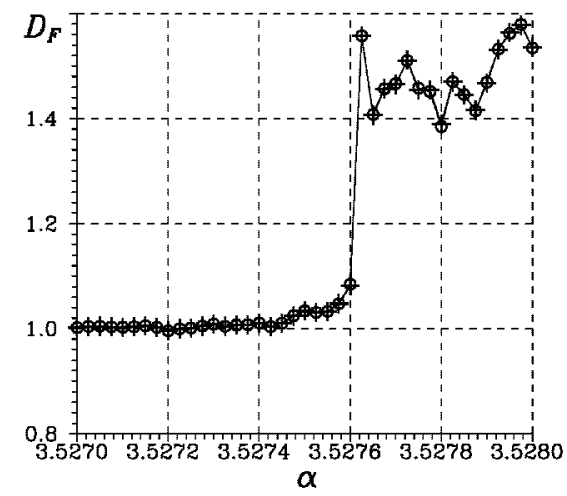

FIGURE 1. Fractal dimension $D_{F}$ as a function of parameter $\alpha$ in the absence ("o") and in the presence (" + ") of noise.

To diagnose the presence of chaos, it is conventional to calculate the largest Lyapunov exponent (LLE) characterizing the evolution of infinitely-small perturbations over an infinitely-large time period. A positive LLE implies that the perturbation grows exponentially, indicating a sensitivity to initial conditions that leads to mixing and thus chaos. The connection between chaos and the sign of the LLE has been strictly proven only for hyperbolic and quasihyperbolic systems [7]. For the nonhyperbolic systems with which we usually deal in practice, including the logistic map (1), there is as yet no rigorous proof of such a connection. In many papers, therefore, in addition to calculating the LLE, authors also explore sensitivity to initial conditions by use of a variety of more direct methods [1,4,8-10]. For example, in $[9,10]$ the evolution of an ensemble of initially closely spaced trajectories $\left\{x_{n}^{i}, \phi_{n}\right\}$, characterized by the same phase of the external force, is considered. If the distance between trajectories tends to zero asymptotically as $t \rightarrow \infty$ (achieves the 
size of attractor), sensitivity is absent (present), and the attractor is nonchaotic (chaotic). We have used this method, in addition to calculation of the LLE, to diagnose the types of attractor in (1). To describe the evolution of an ensemble of trajectories we have studied the dispersion $S(n)[9]$ :

$$
S(n)=\left[\frac{1}{N} \sum_{i=1}^{N}\left(x_{n_{i}}^{i}-\left\langle x_{n}^{i}\right\rangle\right)^{2}\right]^{1 / 2} ;
$$

here $N$ is the number of trajectories in the ensemble, and " \langle\rangle " indicates an ensemble average. A set of points $\left\{x_{0}^{i}\right\}$ at time $t=0$ is located within an interval of length $l=0.0001$, at the center of which lies a point belonging to the attractor. The sensitivity to initial conditions may then be characterized by e.g. the maximal dispersion $S_{\max }$. This has been calculated over 1000000 iterations, following a relaxation time of similar duration.

In Fig. 2 the LLE and $S_{\max }$ as functions of $\alpha$ are shown by the circle data. It can be seen (Fig. 2(a)) that, for $\alpha<3.52792$, the LLE is negative indicating an absence of sensitivity to initial conditions. Consistent with $[4,11]$ and the results shown in Fig. 1 and Fig. 2(a), we confirm that in the noise-free $(D=0)$ map: for $\alpha<3.52760$ the torus is an attractor of the map (region 1); that for $3.52760<\alpha<3.52792$, an SNA arises (region 2); and that for $\alpha>3.52792$ a strange chaotic attractor exists (region 3). However, the calculated $S_{\max }$ demonstrates (Fig. 2(b)) an absence of sensitivity to initial conditions - including the region of positive LLE.
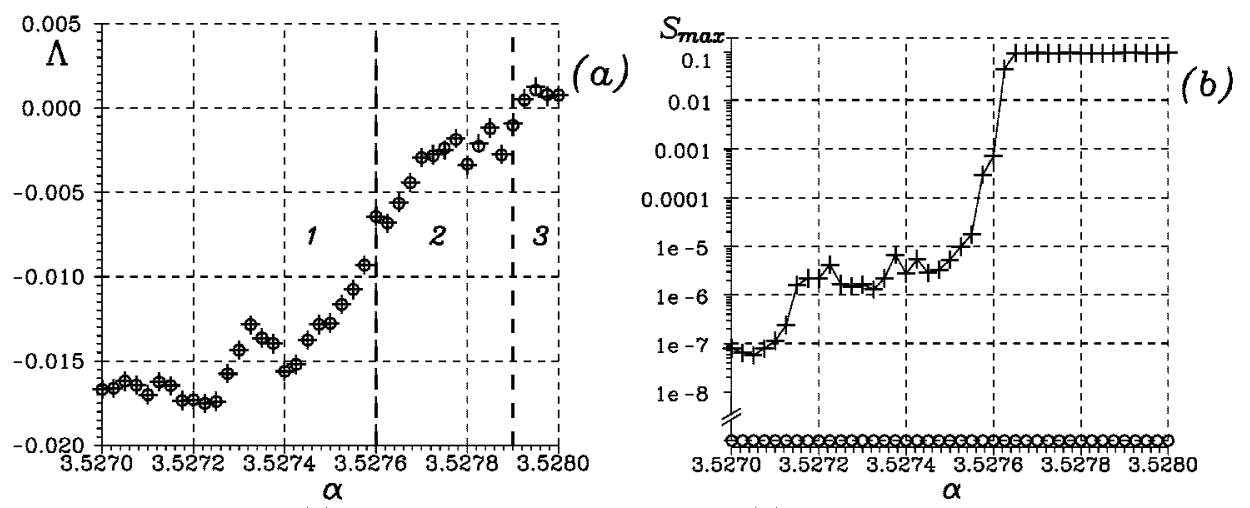

FIGURE 2. LLE $\Lambda$ (a) and maximal dispersion $S_{\max }$ (b) as functions of parameter $\alpha$ in the absence ("o") and in the presence ("+") of noise.

To account for these apparent contradictions, we have analyzed the time evolution of an infinitely-small perturbation. Its magnitude $\epsilon(n)$ at iteration $n$ is defined by $\epsilon(n)=\epsilon_{0} \exp \left(\lambda_{s}(n)\right)$; here $\epsilon_{0}$ is the initial perturbation at $n=0$; and $\lambda_{s}(n)$ is the sum of local Lyapunov exponents during the time $n$ :

$$
\lambda_{s}(n)=\sum_{i=0}^{n} \log \left|\alpha\left(1+\beta \cos \phi_{i}\right)\left(1-2 x_{i}\right)\right| .
$$


The quantity $\exp \left(\lambda_{s}(n)\right)$ is defined as the factor by which the initial perturbation $\epsilon_{0}$ has changed in size at the $n$th iteration. Note that the LLE $\Lambda$ and $\lambda_{s}(n)$ are connected by $\Lambda=\lim _{n \rightarrow \infty} \lambda_{s}(n) / n$. In Fig. 3(a) the evolution of $\lambda_{s}(n)$ is shown for different types of attractor. For the torus and SNA, the initial perturbation decreases, whereas for chaos it increases as $t \rightarrow \infty$. It can be seen (insert in Fig. 3(a)) that, for all types of attractors, the size of the perturbation alternately decreases and increases. Thus perturbations of trajectories in the torus and SNA regimes are not necessarily either decreasing or increasing at any given moment of time. On the other hand, in the region of the chaotic attractor, the perturbation can decrease locally.
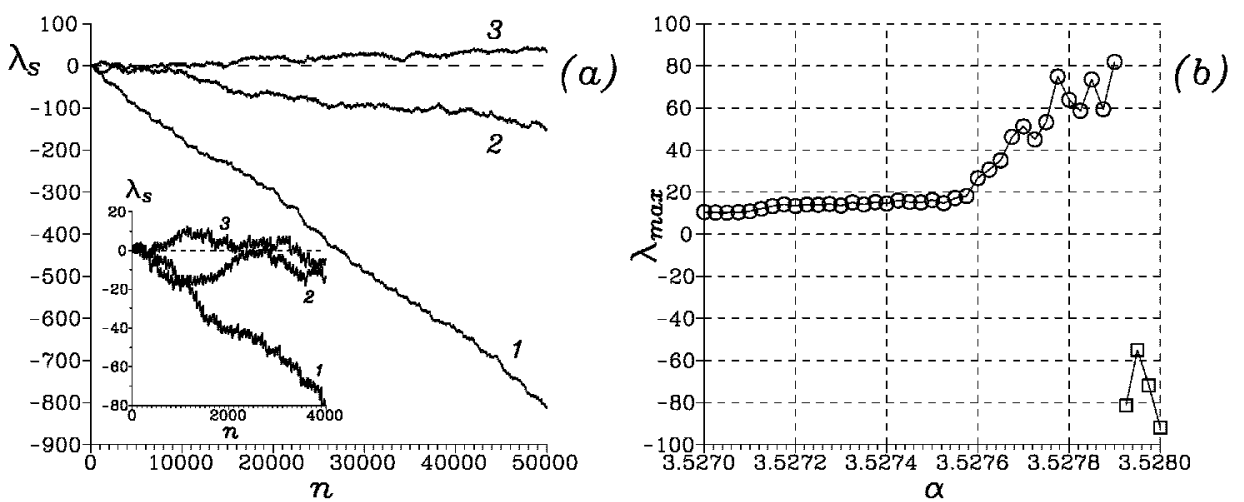

FIGURE 3. (a) Evolution $\lambda_{s}(n)$ for different types of attractors: 1 - torus, $\alpha=3.52740 ; 2-$ SNA, $\alpha=3.52780 ; 3-$ chaotic attractor, $\alpha=3.5280$. The insert is an expansion for small $n$. (b) $\lambda_{\max }$ as function of $\alpha$ for the torus and SNA ("o") and for chaos (" $\square$ ").

We have calculated the maximal factors $\lambda_{m a x x}$ of increase for the torus and SNA, and decrease for chaos. For chaos, the perturbation decreases by more than $\exp (50)$ during some time intervals, i.e. the distance between nearby trajectories of the ensemble can decrease to a value less than the truncation errors. The trajectories must subsequently evolve together because two double-precision numbers differing only in the 17th decimal place cannot normally be distinguished with a computer. For precisely this reason, the maximal distance between nearby trajectories of the chaotic attractor falls to zero, despite their positive LLE (Fig. 2(a)). In the region of the torus (Fig. 3(b), circles, $\alpha<3.52760$ ), the perturbation can increase by $\sim$ $\exp (15)$ times; for the SNA (Fig. 3(b), circles, $3.52760<\alpha<3.52792$ ), it can increase by $\sim \exp (50)$ times. We find that the size of initial perturbation $\epsilon_{0}$ which will not become comparable with the size of the attractor during these time intervals is $\sim 10^{-8}$ (torus), and $\sim 10^{-24}$ (SNA). If the perturbation exceeds these values, then mixing will occur. Recall however (Fig. 3(a)) that perturbations within the torus and SNA regimes tend to zero asymptotically as $t \rightarrow \infty$.

In reality we almost always deal with finite-time perturbations, because the fluctuations in real systems occur continuously. In the presence of fluctuations the 
size of perturbation cannot be less than some value corresponding to the noise intensity [12]. In other words, when we take account of fluctuations, two initially close trajectories will always differ from each other by some value; this is true even for the regular regime. Consequently, within all regimes perturbations must evolve as follows: during some time intervals the perturbation increases, whether mixing or not (dependent on the noise intensity), and during other time intervals the perturbation decreases to a non-zero value defined by the noise intensity.

We now address the question: does weak noise change the geometrical properties of the attractors? We consider noise of intensity $D=10^{-13}$, substantially larger than the truncation errors of $\sim 10^{-17}$. In Fig. 1 the dependence of $D_{F}$ on $\alpha$ is shown (pluses) for the noisy system. It is evident that the geometrical characteristics remain quite unchanged. Next, we return to consider the dynamical properties of the noisy map. We suggest that in region 1 (Fig. 2(a)) the mixing of trajectories will be absent because, for mixing, it is necessary for the initial perturbation to exceed $\sim 10^{-8}$. In region 2 we will observe mixing which must occur where fluctuations exceed $10^{-24}$. So, the behavior of trajectories in regions 2 and 3 must be similar. In general they cannot be distinguished for noise intensities exceeding $10^{-24}$. Hence for $\alpha>3.52760$ in the noisy map we will observe a strange chaotic attractor, because mixing exists and an SNA cannot arise.

To confirm these ideas, we have examined the evolution of an ensemble of trajectories and the behavior of the LLE in the presence of very weak noise. It can be seen (Fig. 2(a)) that the LLE is unaffected by whether there is weak noise in the map or not. The maximum dispersion (Fig. 2(b)) differs from zero everywhere but, for $\alpha>3.52760$, it is comparable in size to the attractor. This suggests a sensitive dependence on initial conditions, i.e. chaos. Note that, for the noise-free map in this range of $\alpha$, we observed (see above) both an SNA and a chaotic attractor.

Thus, weak noise does not influence the fractal structure (strangeness) of an attractor, but it transforms an SNA of the quasiperiodic system into an attractor characterized by a sensitivity to initial conditions, i.e. a strange chaotic attractor, albeit one that is characterized by a negative LLE. This implies that use of the LLE as an indicator of the velocity of perturbation growth gives misleading information about the dynamical properties of attractors in the quasiperiodic system with noise. We have therefore calculated the complexity measure $K_{\sigma}$, which was introduced [12] for the analysis of chaotic systems in the presence of fluctuations. It defines the velocity of divergence of nearby trajectories and is calculated for two trajectories with different noisy realizations. For regular motion, $K_{\sigma}=0$, but $K_{\sigma}$ differs from zero in the chaotic regime. It can be seen (Fig. 4), that the complexity differs from zero in the regions where an SNA and chaos exist in the noise-free system, and that $K_{\sigma}=0$ in the region of the torus. This reinforces our conclusion that, for $\alpha>3.52760$ in the presence of noise, the motion is chaotic.

So it would appear that, for the system considered, the presence of extremely weak noise means that chaos is necessarily associated with strangeness: the SNA seen in the noise-free system ceases to exist once noise is added. We have established similar results for the other systems [2] where an SNA arises near the boundary of a 


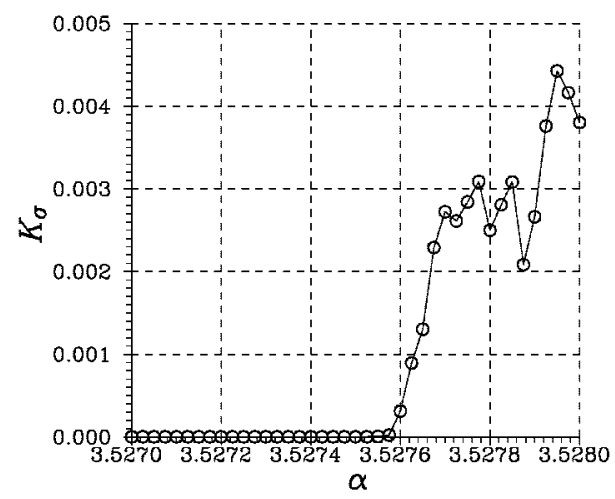

FIGURE 4. Measure of the complexity $K_{\sigma}$ as a function of parameter $\alpha$.

chaotic regime. If, as appears to be the case, these results are generally applicable, and since noise is invariably present in real macroscopic physical systems, we must conclude that in reality SNAs do not arise as the intermediate stage in the evolution from regular to chaotic behavior.

The research was supported by INTAS (grant N 96-0305), and by the Royal Society of London. N.A.Kh and I.A.Kh would like to acknowledge the hospitality of Lancaster University. I.A.Kh gratefully acknowledges support from Max-PlanckInstitute for Physics of Complex Systems in Dresden.

\section{REFERENCES}

1. Grebogi C., Ott E., Pelikan S., and Yorke J.A., Physica 13D, 261 (1984).

2. Ding M., Grebogi C., and Ott E., Phys. Rev. A 55, 2593 (1989).

3. Matsumoto K., and Tsuda I., J. Stat. Phys. 31, 87 (1983); Bulsara A.R., Jacobs E.W., and Schieve W.C., Phys. Rev. A 42, 4614 (1990); Wiesenfeld K., Moss F., Nature 373, 33 (1995); M.Millonas, Fluctuations and Order: The New Synthesis, INLS New York: Springer, 1994.

4. Heagy J.F., and Hammel S.M., Physica 70D, 140 (1994).

5. Prasad A., Mehra V., and Ramaswamy R., Phys. Rev. E 57, 1576 (1998).

6. Farmer J.D., Ott E., Yorke J.A., Physica 7D, 153 (1983); Russel D.A., Hanson J.D., Ott E., Phys. Rev. Lett. 45, 1175 (1980).

7. Pesin J.B., Uspekhi Mat. Nauk 32, 55 (1977).

8. Nicolis C., and Nicolis G., Phys. Rev. A 43, 5720 (1991).

9. Lai Y.-C., Feudel U., and Grebogi C., Phys. Rev. E 54, 6070 (1996).

10. Romeiras F.J., Grebogi C., and Ott E., Phys. Rev. A 41, 784 (1990).

11. Lichtenberg A.L., Liberman M.A., Regular and Stochastic Motion, New-York: Springer-Verlag, 1983.

12. Palladin G., Serva M., and Vulpiani A. Phys. Rev. Lett. 74, 66 (1995). 
Copyright $\odot 2003$ EBSCO Publishing 
Copyright of AIP Conference Proceedings is the property of American Institute of Physics and its content may not be copied or emailed to multiple sites or posted to a listserv without the copyright holder's express written permission. However, users may print, download, or email articles for individual use. 